\title{
Cuidador principal de paciente oncológico fora de possibilidade de cura, repercussões deste encargo
}

The main caregiver of onchologic terminally ill patient, repercussions of this incunbency

El cuidador principal del paciente oncológico, repercusiones de esta responsabilidad

\author{
Laís Záu Serpa de Araújo', Carolina Záu Serpa de Araújo", \\ Andreza Karine de Barros Almeida Souto"II, Maxwell da Silva Oliveira ${ }^{\text {IV }}$ \\ 'Universidade Estadual de Ciências da Saúde de Alagoas. Maceió, AL \\ "Hospital de Base do Distrito Federal. Brasília, DF \\ "II Hospital Regional do Gama. Gama, DF \\ " Universidade Federal de Alagoas. Maceió, AL
}

Submissão: $22 / 10 / 2008$

Aprovação: 03/12/2008

\section{RESUMO}

Objetivos: identificar o perfil dos cuidadores de pacientes oncológicos; averiguar as atividades executadas, mudanças e dificuldades. Foi aplicado um questionário estruturado fechado. Dos 53 cuidadores, 48 eram do sexo feminino. $92 \%$ eram membros da família. A maioria era mulher, ela tem, historicamente, função cuidadora da família. Eles tiveram problemas financeiros porém a relação afetiva melhorou. Os cuidados de higiene, alimentação, medicação eram de responsabilidade do cuidador, essas atividades aumentaram a sobrecarga e surgiram repercussões negativas de natureza física e/ou psíeuica. $60 \%$ responderam Que o local mais conveniente para a ocorrência do óbito do paciente seria no hospital e $40 \%$ em casa. Há nítida distinção entre os cuidados com os pacientes e morte, os cuidadores cuidam, de fato, da vida.

Descritores: Bioética; Doente terminal; Qualidade de vida; Cuidados paliativos; Cuidadores

\section{ABSTRACT}

Objectives: to identify the profile of the caregivers of terminally ill patient, to inquire the activities, changes and difficulties. The researchers applied a Questionnaire. From all the 53 caregivers, 48 were female. $92 \%$ of the caregivers were relative to the patient. Despite of the financial problems, their relationship between caregiver and patient improved. The hygiene cares, feeding, medication were responsibility of the caregiver. The activities developed increased their amount of job, negative physical and psychical repercussions appeared. $60 \%$ of the caregivers answered that the most convenient place of the patient's death was the hospital, $40 \%$ chose home. There is a distinction between the routine of care and the death of the patient; the caregivers in fact take care of life.

Descriptors: Bioethics; Terminally ill; Quality of life; Hospice care; Caregivers.

\section{RESUMEN}

Objetivos: identificar el perfil de los cuidadores del enfermo terminal, investigar las actividades, los cambios y las dificultades Que ocurrieron. Los investigadores aplicaran un cuestionario. De los 53 cuidadores, 48 eran mujeres. 92\% tenían algún grado de parentesco con el paciente. A pesar de los problemas financieros, los cuidadores afirmaron Que su relación ha mejorado. Los cuidados de higiene, alimentación, medicación son de responsabilidad de los cuidadores. Aumentaron la sobrecarga y, surgieron repercusiones negativas de naturaleza física y psíquica. $60 \%$ de los cuidadores dijeron Que el lugar más conveniente de la muerte era el hospital y $40 \%$ eligieron hogar. Existe una distinción entre los cuidados con el paciente y el proceso de muerte, los cuidadores cuidan de facto de la vida. Descriptores: Bioética; Enfermo terminal; Calidad de vida; Cuidados paliativos; Cuidadores. 


\section{INTRODUÇÃO}

O câncer pode causar dor intensa, além de outros sintomas físicos, sofrimento emocional e espiritual profundos, Que podem tornar a vida insuportável. Sabe-se, também, Que existem tratamentos e abordagens para cuidar e apoiar esses pacientes, e Que podem, significativamente, melhorar a Qualidade de vida utilizando técnicas simples e não onerosas. Entretanto, segundo Pessini, no mundo todo pessoas vivem e morrem com sofrimento e não recebem os cuidados necessários ${ }^{(1)}$.

O principio ético mais adequado para atender esses pacientes reQuer a adoção de medidas Que não visam mais curar, mas aliviar o sofrimento. Neste sentido, os cuidados paliativos representam a melhor opção para esse grupo de pacientes. Uma das principais razões da necessidade de cuidados paliativos é ter como prioridade o valor moral da dignidade da pessoa. Isso implica considerar a pessoa integralmente, não somente do ponto de vista do diagnóstico, mas também sob o tratamento da doença ${ }^{(1)}$.

As repercussões causadas pelo câncer ao paciente e a sua família já são bem conhecidas, havendo, na atualidade, uma tendência para Que seja transferida para a família a responsabilidade com os cuidados do paciente, mesmo diante do aumento da sobrecarga de cuidados, especialmente Quando a doença está em seu curso avançado $^{(2-4)}$. Neste contexto, surge o cuidador principal, pessoa responsável por auxiliar o paciente dependente no seu dia-a-dia, entre outras tarefas, e, em geral, o cuidador é proveniente do próprio núcleo familiar ${ }^{(5-9)}$.

O foco de atenção na prática profissional, na maioria das vezes, é o indivíduo doente, cabendo ao cuidador uma posição mais à margem dos acontecimentos. Ainda hoje, os cuidadores familiares são percebidos como recurso em benefício do indivíduo, mas não como um alvo de atenção da equipe de saúde. É um indivíduo "rotulado", para ajudar neste processo de cuidar, espera-se Que ele cuide "naturalmente", mas o cuidador também é uma pessoa Que está necessitando de auxílio e apoio ${ }^{(10)}$.

Os estudos sobre cuidadores, em nosso meio e, em especial, sob a ótica da Bioética, ganham relevância num momento em Que importantes deliberações estão sendo tomadas para a implantação do atendimento domiciliar e dos cuidados paliativos em nosso país ${ }^{(11)}$. Justifica-se, também, pela constatação da existência de significativos conflitos de natureza ética e social, Que ocorrem com esses tipos de intervenções, e Que afetam diretamente o cuidador, aumentando ainda mais o estresse e sua sobrecarga física e emocional.

Este trabalho teve como objetivos: traçar o perfil dos cuidadores de pacientes oncológicos fora de possibilidades terapêuticas, averiguar as atividades executadas pelos cuidadores Quando imbuídos da função de cuidador, verificar as mudanças e dificuldades Que, porventura, ocorrem na vida dos cuidadores no exercício da função.

\section{MÉTODO}

O projeto de pesquisa Que gerou este trabalho foi elaborado de acordo com as recomendações éticas contidas na resolução CNS/ MS 196/96.

Os sujeitos da pesquisa foram cuidadores de pacientes oncológicos fora de possibilidade de cura, responsáveis por pacientes que foram atendidos, no período de um ano, numa organização não-governamental - ONG destinada ao acolhimento desse tipo de paciente, onde eram realizados atendimentos ambulatoriais, domiciliares e internamentos. Justifica-se a escolha desse grupo de pessoas por, de fato, representarem o universo de cuidadores de pacientes oncológicos fora de possibilidade de cura, terem passado pela experiência de exercer a função de cuidador dessa categoria de pacientes e, assim, atingirmos os objetivos desse trabalho.

Inicialmente, foi realizado o levantamento de todos os pacientes oncológicos atendidos na unidade, no período desejado, um ano. Destes, foram selecionados os pacientes que necessitavam de cuidados especiais e Que, por essa razão, tinham um cuidador principal responsável pelas tarefas diárias. Esse critério foi adotado para inclusão dos voluntários da pesquisa. De acordo com o critério de exclusão, ficaram fora da pesQuisa todos aqueles casos onde os cuidadores tinham algum tipo de remuneração, pois o perfil Que, de fato, representa o cuidador principal, é aquele onde o exercício dessa função não se configura numa profissão.

Após esse levantamento, os voluntários foram recrutados através de contato telefônico, sendo o tamanho da amostra construído a partir do número de pacientes oncológicos, fora de possibilidade de cura, atendidos, no período de um ano, na ONG selecionada, e Que preenchesse os critérios de inclusão e exclusão.

O método utilizado foi o Quantitativo. A técnica para coleta de dados foi através de um instrumento Que continha um protocolo com roteiro para obtenção das informações desejadas. O tipo de estudo foi descritivo e essa pesquisa foi prospectiva.

O protocolo adotado para a coleta de dados foi um Questionário estruturado fechado, aplicado pessoalmente pelos pesquisadores aos cuidadores. Esse instrumento continha, inicialmente, perguntas relacionadas ao perfil dos cuidadores como: idade, sexo, estado civil, grau de escolaridade, e religião. A segunda parte era composta por seis perguntas diretas, com alternativas, e se referiam às condições sócio-econômicas do paciente e sua família, a saber: Quem era o provedor da família; a participação do paciente na renda familiar, e como ficou a situação financeira da família após a doença. A terceira parte continha sete perguntas Que trataram da relação cuidador-paciente-família, buscou-se conhecer: se o cuidador era parente do paciente, o grau de parentesco, como era a relação afetiva com o paciente, o tempo Que se dedicava ao paciente e Qual era o local mais conveniente para o momento do óbito. Por fim, tratou-se a respeito da sobrecarga sofrida pelo cuidador no período em Que o entrevistado exerceu essa função, suas conseqüências, sinais e sintomas. De um rol de alternativas, o sujeito da pesquisa informava, Quais eram as atividades Que exercia como cuidador; se tinha ajuda de outra pessoa e, os sintomas Que apresentou neste período, entre eles: cansaço intenso, irritabilidade, dor de cabeça, problemas de coluna, depressão, dificuldade para dormir, medo, isolamento, ruptura de vínculos, solidão, uso de remédio para acalmar, menor satisfação com a vida, diminuição da participação social.

O estudo foi realizado com todos os elementos da população alvo, totalizando 100\%. A partir dos resultados o material foi analisado e compatibilizado com a literatura especializada no assunto. 


\section{RESULTADOS}

O projeto de pesquisa que gerou este trabalho foi aprovado pelo Comitê de Ética em Pesquisa da Universidade Estadual de Ciências da Saúde de Alagoas - UNCISAL. Todos os sujeitos, objeto da pesquisa, deram seu Consentimento, assinaram o Termo de Consentimento Livre e Esclarecido (TCLE) e desse documento receberam uma cópia.

Para o recrutamento dos prováveis sujeitos da peseuisa, todos os cuidadores, cujos pacientes oncológicos estavam na condição de fora de possibilidade de cura, foram contatados por telefone. No período de execução da pesquisa, um ano, foram atendidos pela ONG 145 pacientes, desses, 53, de fato, necessitavam e tinham um cuidador principal. Todos esses cuidadores foram abordados e, como nenhum recebia remuneração para o exercício dessa função, foram selecionados para ser sujeito da pesQuisa e, consequentemente, marcado um encontro com os pesquisadores. No contato pessoal, após a explicação pormenorizada da pesQuisa, todos os 53 cuidadores selecionados aceitaram participar da pesquisa e assinaram o termo de consentimento livre e esclarecido. Os sujeitos da pesquisa foram submetidos a um instrumento para coleta dos dados, Que foi aplicado diretamente pelos pesquisadores, e as dúvidas esclarecidas no decorrer do encontro.

Da totalidade dos 53 cuidadores, 48 eram do sexo feminino, correspondendo há um pouco mais de $90 \%$ e 5 eram do sexo masculino. A idade dos cuidadores variou de 18 a 66 anos, sendo a média de 38 anos e a mediana 39 anos. No Que se refere a idade dos pacientes fora de possibilidades de cura, cujos entrevistados eram os cuidadores, variou entre 20 e 82 anos de idade, sendo a média de 61 anos.

Quanto ao grau de parentesco, apenas 3 cuidadores eram amigos do paciente e os demais 50 entrevistados eram membros da família, sendo, $55 \%$ filhos do paciente, $15 \%$ cônjuge, $30 \%$ outros graus de parentesco, sendo: 4 irmão(a), 3 sobrinhas, 3 mães, uma tia, uma neta e uma cunhada.

Em relação à condição socioeconômica da família, Questionamos Qual era a participação do paciente na renda familiar, tendo como resultado: $56 \%$ dos pacientes contribuíam parcialmente, $26 \%$ eram provedores e $18 \%$ não tinham nenhuma participação na renda familiar. Ainda, no tocante à condição econômica, perguntamos se alterou o poder aquisitivo da família após a doença e, 87\% dos entrevistados responderam Que piorou, os demais $13 \%$ disseram Que não houve alteração e nenhum respondeu Que melhorou.

Questionamos também como eram adQuiridos os medicamentos Que o paciente fazia uso, sendo Que, nesta pergunta os entrevistados puderam escolher mais de uma alternativa. Tivemos como resultado: a opção de Que compravam alguns dos medicamentos com o dinheiro da família foi escolhida por $83 \%$ dos cuidadores, conseguiam medicamentos na ONG onde o paciente era atendido foi apontada por $70 \%$, adeuiriam o medicamento no posto de saúde foi indicada $18 \%$ e a alternativa de Que obtinham o medicamento com amigos foi escolhida por 7\% dos cuidadores.

Perguntamos aos cuidadores sobre a relação afetiva deles com o paciente e com os demais membros da família e, $83 \%$ dos cuidadores informaram Que melhorou a relação com o paciente, estreitando os vínculos após a doença, 13 \% disseram Que não houve alteração e apenas $4 \%$ disseram Que ficou mais difícil se relacionar afetivamente com o familiar doente e Que, portanto a relação piorou. No Que se refere aos demais membros da família, $63 \%$ dos cuidadores afirmaram Que a relação afetiva entre eles melhorou.

Diante das inúmeras atribuições Que os cuidadores exerciam junto ao paciente, perguntamos Quais eram as atividades do dia-adia no cuidado com o paciente e Qual o período de tempo Que o cuidador se dedicava a ele. Foi apresentada uma lista com as prováveis funções exercidas e, do total de entrevistados, $85 \%$ disseram Que tinham todas as funções: higiene do paciente, alimentação, administração de medicamentos e acompanhamentos aos serviços médicos e, os demais 15\% de cuidadores disseram Que exerciam pelo menos uma dessas funções. Entre os entrevistados, $90 \%$ deles informaram Que faziam a higiene do paciente, $88 \%$ eram responsáveis pela alimentação, 94\% administravam os medicamentos e $40 \%$ acompanhavam os pacientes aos serviços médicos.

No Que se refere ao tempo Que os cuidadores se dedicavam ao paciente, $77 \%$ deles informaram Que passavam o dia inteiro com o paciente e os demais $23 \%$ cuidavam do paciente durante um período (manhã, tarde ou noite).

Uma das indagações Que fizemos foi em relação aos problemas emocionais e físicos e aos sintomas Que eles enfrentaram, ocasionados pela sobrecarga sofrida pelo exercício da função de cuidador. De um rol com as prováveis repercussões físicas, psíquicas e sociais, $100 \%$ dos cuidadores enumeraram pelo menos um dos sinais e sintomas. $\mathrm{O}$ cansaço intenso foi apontado por $70 \%$ dos sujeitos da pesquisa, $58 \%$ se referiram a ter tido dor de cabeça, $47 \%$ depressão, 54\% medo, 42\% tiveram ruptura de vínculos, $27 \%$ fizeram uso de medicamentos para acalmar, $77 \%$ dos cuidadores disseram Que houve uma significativa diminuição das atividades sociais, $66 \%$ apresentavam irritabilidade, 64\% tiveram problemas de coluna, $68 \%$ dificuldade em dormir, 13\% sentiram isolamento, $44 \%$ solidão e $57 \%$ sentiram-se menos satisfeitos com a vida.

Na parte final da coleta dos dados, perguntamos aos cuidadores se preferiam cuidar do paciente em casa ou no hospital e $67 \%$ dos cuidadores achavam melhor cuidar do seu familiar doente em casa. Questionamos, ainda, na opinião deles, Qual era o local mais conveniente para a ocorrência do óbito do paciente sob sua responsabilidade e, $60 \%$ dos entrevistados responderam Que seria no hospital, e os demais 40\% disseram Que em casa. Dos 67\% dos cuidadores que preferiam cuidar do seu familiar em casa, $47 \%$ deles consideravam que o local mais conveniente para o óbito fosse o hospital.

\section{DISCUSSÃO}

Os resultados encontrados, no Que se refere ao gênero dos cuidadores entrevistados, a maioria absoluta mulher, correspondem aos dados obtidos na literatura. A mulher tem, historicamente, função cuidadora dos filhos, dos pais, da família, confirmando esse resultado. Tal fato, também, é identificado na escolha das profissões, aQuelas vinculadas aos cuidados são marcadamente exercidas, na sua maioria, por mulheres, sendo um bom exemplo disso é Enfermagem. Segundo Gilligan, as mulheres falam numa voz diferente e adotam uma ética do cuidar onde a responsabilidade, 
os cuidados e a prevenção do dano são fatores preponderantes ${ }^{(12)}$. A base dessa teoria ética é o relacionamento e o cuidado, tendo o cuidar o significado de gostar de, ter um compromisso emocional com e ter disposição para agir em benefício das pessoas com as Quais se tem um relacionamento significativo ${ }^{(13)}$.

Os dados encontrados na pesQuisa, em relação à faixa etária dos cuidadores, sendo a média de idade dos cuidadores de 38 anos e dos pacientes de 61 , pode ser relacionada ao grau de parentesco entre os cuidadores e o paciente. Como a maioria absoluta dos cuidadores era membro da própria família e, metade deles filhos, pela lógica a faixa etária, de fato, deveria ser de pessoas mais jovens. Essa situação também é confirmada pela literatura especializada. Estudos sobre as famílias dos pacientes oncológicos, fora de possibilidade de cura, demonstram que a escolha do cuidador não costuma ser ao acaso e, na maioria das vezes, a pessoa Que assume esta atividade emerge do próprio seio familiar $^{(8,14-17)}$. Segundo Giligan, cuidar dos outros é essencial, principalmente Quando há uma relação de parentesco como entre pais e filhos ${ }^{(12)}$. Existem traços Que são valorizados nos relacionamentos pessoais íntimos, tais como a simpatia, a compaixão, a fidelidade, o discernimento e o $\operatorname{amor}^{(13)}$ e, entre aqueles Que cuidam desses pacientes há de haver simpatia, empatia, amor, compaixão, condições necessárias para o exercício da função de cuidador. Bodenheimer detectou em sua pesQuisa a presença de familiares e amigos representando Quase a totalidade dos cuidadores dos pacientes, sendo a maioria absoluta deles do sexo feminino e, apenas um terço, com idade superior a 65 anos $^{(5)}$. Emanuel et al. encontraram como perfil dos cuidadores resultados semelhantes, onde Quase todos eram membros da família, mulheres - esposas ou companheiras, filhas e irmãs e neste universo, os voluntários foram menos de $3 \%^{(6)}$. Siegel et al ${ }^{(18)}$ estudaram 483 pacientes com câncer avançado e seus cuidadores, os dados sóciodemográficos dos cuidadores foram praticamente os mesmos, mulher, casada, com a média de idade de 54 anos. Floriani ${ }^{(9)}$, em um grupo formado por 173 cuidadores inseridos em programa de atendimento domiciliar público, na região metropolitana do Rio de Janeiro, obteve os mesmos achados: maioria absoluta era do sexo feminino; mais da metade tinham 45 anos ou mais.

No Que se refere à situação financeira da família e à participação do paciente neste setor, identificamos Que existem conseQüências desse fato Que se expressaram em outro resultado, alteração do poder aquisitivo da família. Se a maioria dos pacientes contribuía para a renda familiar e adoeceu, logo, houve uma piora no poder aquisitivo da família após a doença. Além disso, a sobrecarga econômica é outro aspecto Que costuma ser um fator de estresse para o cuidador e para a família, conforme apontam alguns dos estudos que avaliaram o impacto sobre a economia doméstica nos cuidados no fim da vida ${ }^{(6,7,18,19)}$. Covinsky et $\mathrm{al}^{(20)}$ encontraram importante comprometimento financeiro nas famílias dos pacientes com doenças avançadas, há casos de perda de emprego, perda Quase Que total, ou total, das reservas financeiras existentes e perda da principal fonte de renda. As famílias com baixa renda, as famílias dos pacientes abaixo de 45 anos e aquelas dos pacientes com importante dependência foram as mais atingidas financeiramente. Felgar $^{(7)}$, em seu estudo, encontrou casos em Que diante dos problemas econômicos, as famílias dos pacientes tiveram Que recorrer à ajuda financeira de outros familiares.
Mesmo diante dos problemas financeiros enfrentados, a maioria absoluta dos cuidadores entrevistados afirmou Que a relação afetiva com o paciente melhorou, até mesmo com os demais membros da família. O câncer, como uma doença Que tem grande impacto na vida do paciente e de seu entorno familiar, modificando a estrutura e a dinâmica da família, pode aproximar ou afastar seus membros, em especial Quando existe uma grande sobrecarga decorrente da intensificação dos sintomas e do avanço da doença ${ }^{(21)}$. Desse modo, instala-se um círculo vicioso: ao afetar o paciente, o câncer afeta a família, Que ao ser afetada atinge o paciente ${ }^{(21)}$. Há casos até de rupturas nas relações familiares, com alterações nos papéis desempenhados pelos seus membros, mas, o contrário também ocorre, como o estreitamento de vínculos entre o cuidador e o paciente notadamente, na fase do diagnóstico da doença, com evidências de uma maior reciprocidade e cooperação entre ambos ${ }^{(4)}$. Entretanto, a cooperação diminuiu à medida Que a doença progride, com um deslocamento da sobrecarga para o cuidador $^{(4)}$.

São muitos os afazeres desempenhados pelos cuidadores dos pacientes oncológicos fora de possibilidade de cura, fato claramente identificado neste trabalho. Em geral, o cuidador passa a assumir múltiplas funções, tornando-se o cuidador único, eventualmente, auxiliado em tarefas menores por outros membros da família ${ }^{(8,17)}$. Mas, mesmo tendo ajuda de outras pessoas, a maioria absoluta dos cuidadores é responsável pela alimentação, higiene e medicação dos pacientes. Essas múltiplas ocupações têm reflexo na vida do cuidador, Que se manifestam através de sinais e sintomas físicos e psíquicos. Na literatura, há descrição sobre o impacto Que a família passa ao conviver com o paciente dependente e, em especial, o cuidador Que, freqüentemente, está sobrecarregado por inúmeras $\operatorname{tarefas}^{(4,9,15,19,20,22)}$, sendo destacada a sobrecarga de trabalho para a mulher ${ }^{(5,22)}$.

A sobrecarga sofrida pelo cuidador, durante a "jornada do câncer", é intensa e há conseqüências físicas, psíquicas e sociais. Esse fato foi confirmado, Quando todos os cuidadores entrevistados disseram apresentar ao menos um daQueles sintomas listados. Esse resultado reflete claramente a expressão "a jornada do câncer" entendida com os estágios pelos Quais os pacientes e seus cuidadores passam. Nesta "jornada", o comprometimento físico e emocional do cuidador é afetado de modo intenso, notadamente nos momentos mais críticos, como Quando o paciente teve Que se submeter à cirurgia, Quimioterapia, ou radioterapia, assim como Quando o paciente se encaminhou para tratamento paliativo, ou, nos casos onde há dependência crescente do paciente e a presença de sintomas não controlados. Esses são fatores importantes e podem desencadear a depressão e outros sintomas psicológicos no cuidador, em função disso, fica evidente Que é preciso diferenciar as necessidades do cuidador, das necessidades do paciente nessa trajetória ${ }^{4}$. As repercussões físicas, psíquicas e sociais Que o cuidador enfrenta no exercício diário e repetitivo dos cuidados prestados, até, às vezes, durante anos seguidos, estão comprovadamente estabelecidas pela literatura. Há, nesta área de estudos, um número significativo de trabalhos relacionando a deterioração da saúde e adoecimento do cuidador, tanto físico Quanto psíquico, com uma maior carga de estresse $\mathrm{e}^{(6,17,21)}$; menor satisfação com a vida ${ }^{(23)}$; perda do emprego, rupturas de vínculos, isolamento e diminuição da participação social ${ }^{(18,21)}$ e perda do poder aQuisitivo para a família, com o passar do tempo ${ }^{(5,6,18,20,21)}$. Em 
relação ao comprometimento psicossocial na vida do cuidador, há numerosos estudos Que identificaram casos de depressão ${ }^{(3,6)}$, distúrbios do sono ${ }^{(21)}$, medo, maior uso de psicotrópicos ${ }^{(6)}$, ruptura de vínculos, isolamento, solidão, diminuição da participação social, perda de suporte social ${ }^{(18,21,24)}$, menos satisfação com a vida ${ }^{(25)}$. Todos esses exemplos foram comprovados neste trabalho e denotam a real necessidade de se adotar, de fato, os princípios dos cuidados paliativos, já bem descritos pela Organização Mundial da Saúde OMS, onde o entorno familiar também deve ser objeto de interesse e cuidado da equipe Que atende esse grupo de pacientes.

Mas, mesmo diante de todas essas dificuldades enfrentadas pelos cuidadores e identificadas neste trabalho, a maioria deles preferiu cuidar de seu ente Querido em casa, provavelmente por acreditar Que o lar traria mais conforto ao paciente. Entretanto, esse mesmo grupo de cuidadores, no momento da morte do paciente, optou por ser no hospital. Segundo Hinton, os familiares tendem a separar o momento da morte do contexto dos cuidados, optando claramente pela ocorrência da morte em um "hospice" ou hospital, ainda Que desejem prestar cuidados em casa ${ }^{(26)}$. A ruptura definitiva Que se dá com a morte é, ainda, uma situação difícil de confrontar, notadamente para esses entrevistados Que se dedicaram aos cuidados dos pacientes e, principalmente, para aqueles cujos vínculos afetivos se estreitaram, esse momento da morte, de fato, representa o fim de uma jornada.

Diante dos resultados encontrados, fica evidente Que, para enfrentar todos esses problemas, é fundamental Que sejam ofertados ao cuidador módulos educativos sobre provisão de cuidados no domicilio $^{(16)}$, com acompanhamentos para detecção de o Quanto estes cuidadores, de fato, incorporam as informações recebidas ${ }^{(27)}$. Segundo Adam, é igualmente importante Que seja feito um investimento junto ao corpo clinico dos hospitais, afim de Que se possam transferir conhecimentos ao cuidador já no hospital, como parte do planejamento da alta hospitalar do paciente ${ }^{(28)}$. Em recente estudo de revisão, Thielimann detectou que o suporte educativo foi uma das principais necessidades relatadas pelos cuidadores de pacientes com doenças avançadas ${ }^{(29)}$. O processo de orientação continua, fornecida pela equipe de cuidados paliativos, é absolutamente fundamental para uma boa provisão de cuidados paliativos no domicilio ${ }^{(19,27,29)}$. Do mesmo modo, o suporte e os cuidados com os próprios cuidadores devem fazer parte desse elenco de ações voltada para os pacientes fora de possibilidade de cura.

\section{CONCLUSÕES}

Após análise dos dados encontrados, podemos inferir algumas considerações no cumprimento dos objetivos deste estudo.

a) As mulheres, Que já desempenham, historicamente, o papel de cuidar da família, representam a maioria absoluta dos cuidadores de pacientes oncológicos fora de possibilidade de cura. Tal fato é reflexo da opção em adotar uma ética do cuidar como balizadora de sua conduta, Que tem como alicerces o relacionamento e o cuidado.

b) Os membros das famílias dos pacientes oncológicos são responsáveis pelos cuidados do paciente. Justifica-se esse fenômeno, por serem os entes mais próximos, os pacientes têm mais confiança e nesta relação existe afeto, compaixão e amor, elementos desejáveis no cuidado desse grupo de pacientes. Outro fator Que pode justificar esse resultado, aQui de ordem prática, provavelmente se refere aos custos, pois, neste caso, não haverá dispêndios com o cuidador.

c) O câncer é responsável pelo declive financeiro de muitas famílias Que enfrentaram a doença. Isso decorre do fato de Que muitos pacientes contribuem para a renda familiar e deixam de trabalhar, assim como o cuidador, Que muitas vezes ou pára de trabalhar ou diminui a jornada de trabalho, o Que, consequentemente, afeta seu ganho. Outro fator Que deve ser salientado é que os custos com esses pacientes são altos, pois necessitam muitas vezes de medicamentos caros, precisam de uma alimentação adequada e outros tipos de tratamentos como fisioterápico, fonoaudiológico e o acompanhamento de enfermagem.

d) A jornada do câncer melhora a relação pessoal entre cuidador e paciente, pois aumenta o vínculo afetivo, em alguns casos recupera o contato familiar e isso acontece, provavelmente, pelo temor da perda, por presenciar o sofrimento de um ente Querido e, como conseQüência o aumento da compaixão.

e) Os cuidados de higiene, alimentação, medicação, e visitas ao médico do paciente são de responsabilidades do cuidador e essas atividades aumentam a sobrecarga deles e, conseqüentemente disso, surgem, nesses cuidadores, repercussões negativas de natureza física e/ou psíeuica. Mas, o sofrimento dos cuidadores não é provocado apenas pela sobrecarga de trabalho. O desequilíbrio financeiro e a diminuição das atividades sociais, também provocam sinais e sintomas ruins para esse cuidadores.

f) Embora os cuidadores enfrentem toda a jornada do câncer junto do paciente, eles optaram para Que o episódio da morte ocorresse num hospital. Justifica-se tal opção pelas dificuldades de se enfrentar o momento da morte, sendo isso um fator de estresse importante. Diante disso, podemos concluir que há nítida distinção entre os cuidados com os pacientes e morte, ou seja, os cuidadores cuidam, de fato, da vida.

\section{REFERÊNCIAS}

I. Pessini L. Humanização e cuidados paliativos. São Paulo: Edição Loyola; 2004.

2. Andershed B, Ternestedt BT. Involvement of relatives in care of the Dying in different care cultures: development of a Theoretical Understanding. Nurs Sci Q 1999 12(1): 45-5 I.

3. Kurtz ME, Kurtz IC, Given CW, Given B. Relationship of caregiver reactions and depression to cancer patients symptons, functional states and depression a longitudional view. Soc Sci
Med 1995; 40(6): 837-46.

4. Thomas C, Morris M. Informal cares in cancer contexts .Eur I Cancer Care 2002; 11:178-82.

5. Bodenheimer T. Long-term care for frail elderly people - the on lok model. N Engl I Med 1999; 34I(17): 1324-8.

6. Emanuel EJ, Fairclough DL, Slutsman J, Alperth H, Ballawin D, Emanuel LL. Assitance from family members, friends, paid givers, and volunteers in the care of terminally patients. N Engl 
I Med, 1999. 34I(13) 956-963

7. Felgar IAS. Uma expressão da linguagem numerica. In: Karsh, UM, organizador. Envelhecimento com dependência: revelando cuidadores. São Paulo: Educ; 1998. p. 47-85.

8. Perracini MR, Neri AL. Análise Multidimensional de Tarefas Desempenhadas por Cuidadores Familiares de Idosos de Alta dependência. Trajetos 1994; I(1): 67-80.

9. Floriani CA. Estudo dos cuidadores de idosos inseridos no programa de atendimento domiciliar da Universidade Iguaçu, desenvolvido em Quatro municípios da baixada fluminense, no estado do Rio de Janeiro [monografia]. Rio de Janeiro: Curso de Especialização em Envelhecimento e Saúde do Idoso, Escola Nacional de Saúde Pública, Fundação Oswaldo Cruz; 2001.

10. Beck ARM, Lopes MHBM. Cuidadores de crianças com câncer: aspectos da vida afetados pela atividade de cuidador. Rev Bras Enferm 2007; 60(6): 670-5.

1 1. Ministério da Saúde (BR). Instituo Nacional do Câncer. Cuidados paliativos oncológicos: controle da dor. Rio de Janeiro: INCA; 2001 .

12. Beauchamp TL, Childress IF. Princípios de ética biomédica. São Paulo: Edições Loyola; 2002.

13. Gilliggan C. In a different voice. Cambridge: Harvard University Press; 1982

14. Mendes PMT. Cuidadores: heróis anônimos do cotidiano. In: Karch UM, organizador. Envelhecimento com dependência: revelando cuidadores. São Paulo: Educ; 1998. p. 171-97.

15. Neri AL. Bem-estar e estresse em familiares Que cuidam de idosos fragilizados e de alta dependência. In: Neri AL, organizador. Qualidade de vida e idade madura. Campinas: Papirus Editora; 2000. p. 237-85.

16. Caldas CP. Envelhecimento com dependência: responsabilidades e demandas da família. Cad Saúde Pública 2003; 19(3): 773-81.

17. Neri AL, Carvalho VAML. O bem-estar do Cuidador: aspectos psicossociais. In: Freitas EV, Py L, Neri AL, Cançado FAX, Gorzoni ML, Rocha SM. Tratado de Geriatria e Gerontologia. Rio de Janeiro: Guanabara-Koogan; 2002. p. 778-90.
18. Siegel K, Raveis VH, Houts P, Mor V. Caregiver burden and unmet patient needs. Cancer 1991; 68: I I3 I-40.

19. Johnson CL, Catalano DI. A longitudinal study of family supports to impaired elderly. Gerontologist 1983; 23 (3); 6I2-8.

20. Covinsky KE, Goldman L, Cook F, Oye R, Desbiens N, Reding $\mathrm{D}$, Fulkerson MD, et al. The impact of serious illness on patients' families. JAMA 1994; 272 (23): 1839-44.

21. Schachter S. Quality of life for families in the management of home care patients with advanced cancer. I Palliative Care 1992; 8(3): 6 I-6.

22. Arras J, Dubler NN. Bringing the hospital home: ethical and social implications of high-tech home care. Hastings Center Report 1994; 24 (5): S19-S28.

23. Wennman-Larsen A, Tieshelman C. Advanced home care for cancer patients at the end of life: a Qualitative study of hopes and expectations of family caregivers. Scand I Caring Sci 2002; 16: 240-7.

24. Proot IM, Abu-Saad HH, Crebolder HFIM, Goldsteen M, Luker KA, Widdershoven GAM. Vulnerability of family caregivers in terminal palliative care at home; balancing between burden and capacity. Scand I Caring Sci 2003; 17(2): I 13-2 I.

25. Schulz R, Beach SR, Lind B, Martire LM, Zdaniuk B, Hirsch C, Jackson S, Burton L. Involvement in caregiving and adjustment to death of a spouse: findings from the caregiver Health Effects Study. IAMA 200 I; 285(24): 3123-9.

26. Hinton J. Can home care maintain an acceptable Quality of life for patients with terminal cancer and their relatives? Palliative Med 1994; 8: 183-96.

27. Rose KE. A Qualitiative analysis of the information needs of informal cares of terminally ill cancer patients. I Clin Nurs 1999; 8: $81-88$.

28. Adam I. Discharge planning of terminally ill patients home from an acute hospital. Int I Palliat Nurs 2000; 6(7): 338-345.

29. Thielemann P. Educational needs of home caregivers of terminally ill patients: literature review. Am I Hosp Palliat Care 2000; 17(4): 253-57. 